\title{
VIBRATION ANALYSIS OF FLUID-STRUCTURE INTERACTION USING TUNED MASS DAMPERS
}

\section{Ilghar Javanshir}

University of Guilan, University

Department of Mechanical

Engineering,

Rasht, Iran
Campus 2,

\author{
Gholamreza Zarepour \\ University of Guilan, \\ Department of Mechanical \\ Engineering, \\ Rasht, Iran
}

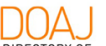

DIRECTORY OF
OPEN ACESS
JOURALS

Crossref

$\mathrm{R} \partial \mathrm{AD} \equiv$

Key words: nonlinear vibration, solid-fluid interaction, vibration absorber, tuned mass dampers, semi-analytical method

\section{Cite article:}

Ilghar, J., \& Gholamreza, Z. [2021]. Bration analysis of fluid-structure interaction using tuned mass dampers. Journal of Applied Engineering Science, 19(2) 318 - 326. DOI:10.5937/jaes0-26043

Online aceess of full paper is available at: www.engineeringscience.rs/browse-issues 


\title{
VIBRATION ANALYSIS OF FLUID-STRUCTURE INTERACTION USING TUNED MASS DAMPERS
}

\author{
Ilghar Javanshir ${ }^{1,}$ Gholamreza Zarepour ${ }^{2 *}$ \\ ${ }^{1}$ University of Guilan, University Campus 2, Department of Mechanical Engineering, Rasht, Iran \\ ${ }^{2}$ University of Guilan, Department of Mechanical Engineering, Rasht, Iran
}

This paper has investigated the semi-analytical analysis of the solid-fluid interaction vibration in the presence of concentrated mass-spring-damper vibration absorber. The nonlinear partial differential equations of motion are derived by considering von Karman-type large deformations and viscoelastic behaviour. Fluid-structure interaction is modelled by using an acceleration coupling model in which a nonlinear Van der Pol oscillator simulates fluctuating nature of the vortex street. The nonlinear equations are discretized via the Galerkin approach, and the obtained equations are numerically solved by applying the Runge-Kutta method. Eventually, the dynamic response, phase plane plots, and variations of maximum amplitude in terms of fluid velocity for different parameters are extracted. The results reveal that utilizing vibration absorber leads to a significant effect on the dynamic characteristics of the system, displaces the lock-in phenomenon, and remarkably reduce the amplitude of the system oscillations.

Key words: nonlinear vibration, solid-fluid interaction, vibration absorber, tuned mass dampers, semi-analytical method

\section{INTRODUCTION}

Fluid-induced vibration which is a nonlinear and self-excited phenomenon occurs in many engineering applications such as aircraft wing and body [1-3], offshore oil and gas pipelines [4-6], marine structures [7], yacht and ship motors [8] and biomedical engineering $[9,10]$. In many of these cases, this type of vibrations can cause fatigue and failure of structures [11], e.g., fatigue caused by fluid-induced has always been a threat to aircraft flight. Therefore, the study of fluid-induced vibration in structures is theoretically and experimentally considered by many researchers [12-14]. Bavil [13] investigates the fluid behavior in a rectangular channel with skewed circular ribs. The operating frequency of the single-sided can be tuned similarly to a tuned mass damper (TMD). In [15] explored the application of shape memory alloy as the damping piece of pounding tuned mass dampers to mitigate the fluctuation of suspended pipes.

Studies show that if the fluid flow velocity is somehow that vortex shedding frequency comes close to the fun- damental natural frequency of the system, large and severe oscillations are created in the structure. This phenomenon, known as the lock-in phenomenon creates large-scale fluctuations, which, if not controlled, can damage the structure or even destroy it. Wu et al. [16] studied the effects of fluid-induced vibration on structures. Kasiri Ghahi and Sanaeirad [17] studied the vibration behaviour of the cylinder at Reynolds number between 144 and 190. Their results show that the amplitude of the oscillations has a significant effect on vortex formation. As the amplitude increases, the vortex lengths and the transverse distance between them decrease, and the longitudinal distance between the vortices varies inversely with the frequency of vibration. In [18-20] investigated the lock-in phenomenon and vortex-induced vibrations in long beams.

The results of their studies showed that the lock-in area is strongly dependent on Reynolds number in long beams. Daniels et al. [21] applied numerical methods to investigate fluid-induced vibration in rectangular cross-section

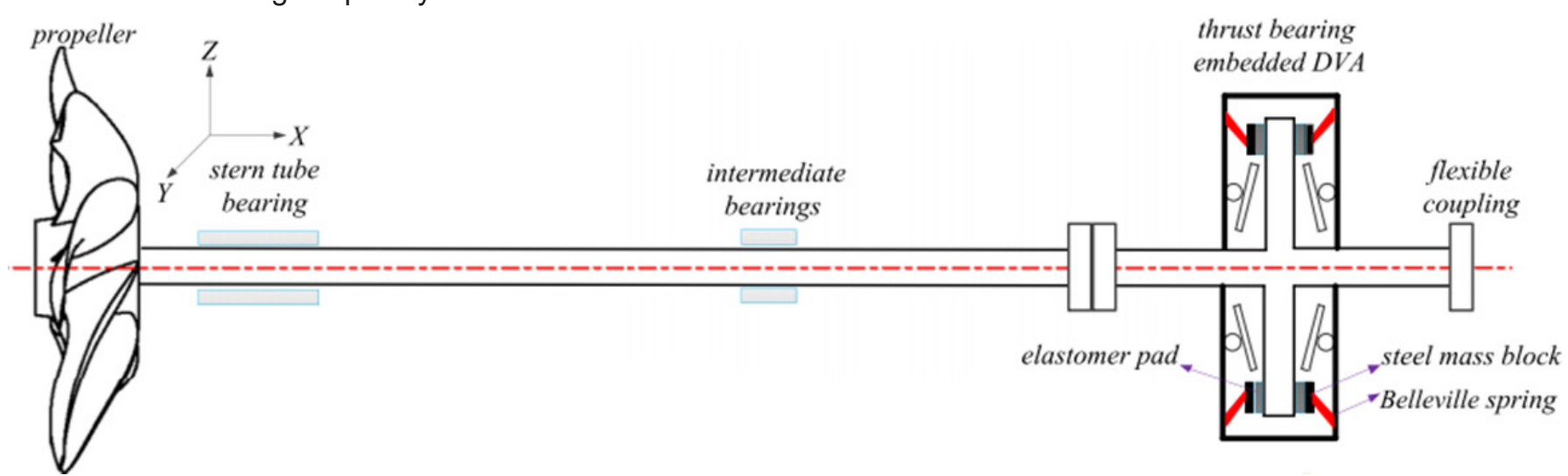

Figure 1: application of a dynamic vibration absorber for control of a marine shafting system [9] 
cylinders. They also studied the behaviour of the system in turbulent flow regions. Marra et al. [22] used empirical tests to improve the analytical model of fluid-induced vibration in rectangular cross-section cylinders. Han et al. [23] investigated the frequency ratio on fluid-induced vibration in cylinders. They simulated cylinder by using a finite element method in the Reynolds range of 200 and analyzed the transient response of the system. Jiang et al. [24] applied numerical methods to investigate fluid-induced vibration in cylinders with a square cross-section.

Vibrations caused by vortices, in which a structure interacts with a fluid flow, and is exposed to vibrations perpendicular to the flow, can ultimately lead to fatigue and failure of the structure. Therefore, studying the applied methods for control and reduction of vibrations caused by vortices is of great importance. Investigations carried out in this field show that tuned mass dampers are inexpensive and straightforward control equipment that can be used in various applications to reduce vibrations. The high performance of TMD absorbers has been reported in multiple applications such as pipelines, towers, beams, and so on. Wang et al. [25] studied the vibration behaviour of the bridge decks using a pendulum massspring-damper system. They used numerical simulations to determine the optimal absorber characteristics, and then experimentally evaluated the designed absorber performance. The results of their study showed that for the absorber with a mass ratio of $2 \%$, the maximum amplitude of the bridge deck oscillations is reduced by $94 \%$. Chen et al. [26] studied the vibration control in a simply supported beam under external harmonic loads, using non-linear mass-spring-damper vibration absorber. Pais and Boote [27] developments a tuned mass damper vibration absorber for yacht structures. They show that the adoption of TMD system could be a suitable solution both at the design stage when for the sake of weight containment heavy structure reinforcements should not be advisable and after construction when any other intervention is not possible.

Reviewing the conducted studies shows that control of fluid-induced vibration in viscoelastic beams by using mass-spring-damper vibration absorber has not been studied yet. Accordingly, the present study semi-analytically investigates the fluid-induced vibration in viscoelastic beams having TMD vibration absorbers. Nonlinear equations are derived by taking into account large deformations and the Kelvin-Voigt viscoelastic model for Euler-Bernoulli beam. After discretization of the equations using the Galerkin method, the effects of different parameters on the dynamic response of the beam is studied.

\section{RESEARCH METHOD}

Fig. 2 displays the geometry of the simply supported viscoelastic Euler-Bernoulli beam. Geometric characteristics and mechanical properties are bending rigidity $E l$, length $L$, cross-section area $A$ and mass per unit volume $\rho$.

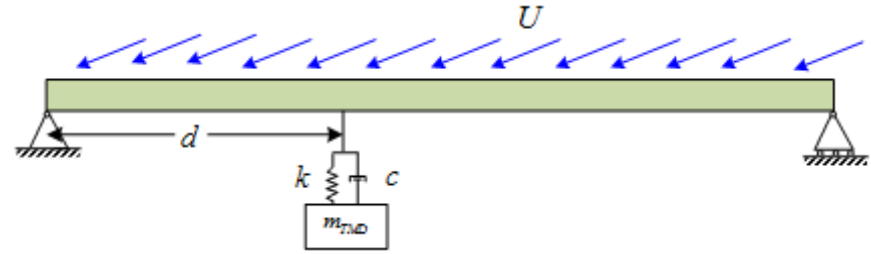

Figure 2: Simply supported viscoelastic Euler-Bernoulli beam

Regarding the transverse vibration of the beam, for the Kelvin-Voigt damping model, which is a two-parameter rheological model, the constitutive relation is expressed as follows:

$\sigma_{x}(x, z, t)=E \varepsilon_{x}+\eta^{*} \frac{\partial \varepsilon_{x}}{\partial t}$

In order to derive the governing differential equation of the beam, the von Kármán type nonlinear strain-displacement relationship of the beam is used. Accordingly, the nonlinear strain associated with the displacement field of the transverse vibration is obtained:

$\varepsilon_{x}=\frac{\partial u}{\partial x}+\frac{1}{2}\left(\frac{\partial w}{\partial x}\right)^{2}$

Considering bending stress $\sigma_{x}$ in Eq. (1), net axial force, $N(x, t)$, and the bending moment, $M(x, t)$, created in the viscoelastic beam is expressed as:

$$
\begin{aligned}
& N(x, t)=\int_{A} \sigma_{x}(x, z, t) \mathrm{d} A=\int_{A} E \varepsilon_{x}+\eta^{*} \frac{\partial \varepsilon_{x}}{\partial t} \mathrm{~d} A \\
& M(x, t)=\int_{A} z \sigma_{x}(x, z, t) \mathrm{d} A=-E I \frac{\partial^{2} w}{\partial x^{2}}-\eta^{*} I \frac{\partial^{3} w}{\partial x^{2} \partial t}
\end{aligned}
$$

Substituting Eq. (2) in Eq. (3), the created axial force is expressed as follows:

$N(x, t)=E A\left[\frac{\partial u}{\partial x}+\frac{1}{2}\left(\frac{\partial w}{\partial x}\right)^{2}\right]+\eta^{*} A\left[\frac{\partial^{2} u}{\partial x \partial t}+\frac{\partial w}{\partial x} \frac{\partial^{2} w}{\partial x \partial t}\right]$

In the following, to derive the nonlinear differential equation, free body diagram of a beam element with the length $d x$ shown in Fig. 3 is used. Here, $M(x, t), Q(x, t)$ and $f(x, t)$ represent bending moment, shear force and the external force applied to the beam, respectively.

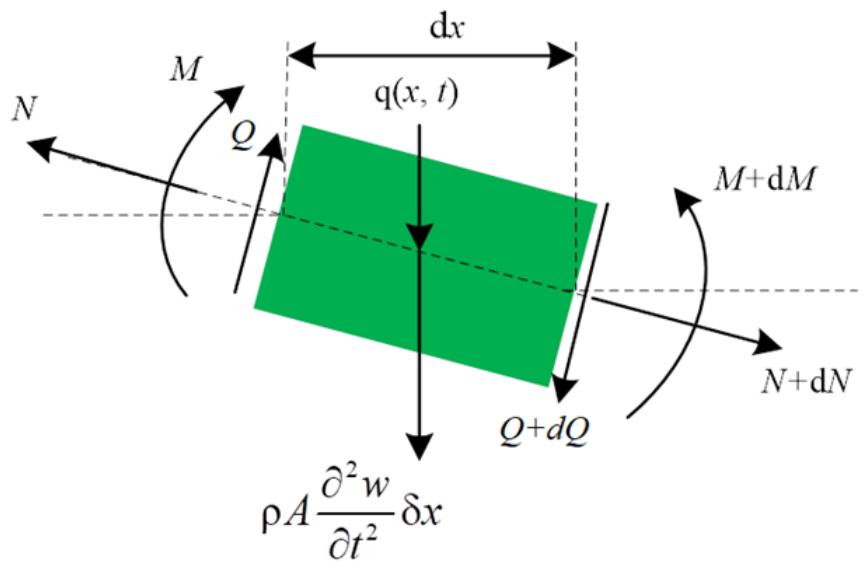

Figure 3: Free body diagram of a beam element 
Given that the inertial force of the beam element is as follows:

$\rho A \mathrm{~d} x \frac{\partial^{2} w(x, t)}{\partial t^{2}}$

writing Newton's second law along the vertical axis, the following equation is obtained:

$\frac{\partial Q}{\partial x}+f(x, t)=\rho A \frac{\partial^{2} w(x, t)}{\partial t^{2}}$

The moment of the forces about point $P$ gives:

$\left(Q+\frac{\partial Q}{\partial x} d x\right) d x-\left(N+\frac{\partial N}{\partial x} d x\right) \frac{\partial w}{\partial x} d x+\frac{\partial M}{\partial x} d x=0$

By neglecting the higher powers of $d x$, the Eq. (8) is simplified as follows:

$Q=-\frac{\partial M}{\partial x}+N \frac{\partial w}{\partial x}$

Using the last equation and Eqs. (3)-(4) the nonlinear governing equation of transverse vibration behaviour of the viscoelastic beam is obtained as follows:

$$
\begin{aligned}
\eta^{*} I & \frac{\partial^{5} w}{\partial x^{4} \partial t}+E I \frac{\partial^{4} w}{\partial x^{4}}+\rho A \frac{\partial^{2} w}{\partial x^{2}} \\
& =f(x, t)+\frac{3}{2} E A\left(\frac{\partial w}{\partial x}\right)^{2} \frac{\partial^{2} w}{\partial x^{2}}+ \\
& +2 \eta^{*} A \frac{\partial^{2} w}{\partial x^{2}} \frac{\partial w}{\partial x} \frac{\partial^{2} w}{\partial x \partial t}+\eta^{*} A\left(\frac{\partial w}{\partial x}\right)^{2} \frac{\partial^{3} w}{\partial x^{2} \partial t}
\end{aligned}
$$

where $f(x, t)$ is the external force applied to the beam, which is divided into two parts: the force applied by the fluid and the pressure applied to the beam by the vibration absorber. Considering the fluid-induced vibration, the external force applied by the fluid consists of two parts: lift force $f_{L}$ and the force caused by hydrodynamic damping applied to the beam in a transverse direction $f_{D}$. According to the studies conducted by Facchinetti et al. [28] and Keber and Wiercigroch [29], these forces are expressed as follows:

$f_{D}(x, t)=-\frac{1}{2} C_{D} \rho_{f} D U \frac{\partial w(x, t)}{\partial t}$

$f_{L}(x, t)=\frac{1}{4} C_{L 0} \rho_{f} D U^{2} q(x, t)$

where $C_{D}$ is damping coefficient which depends on Reynolds number. When the Reynolds number is a subcritical range, i.e. $40<\operatorname{Re}<30 \times 10^{5}$, a low-pressure zone is created behind the structure, in which case the vortex shedding mathematical modelling shows good compatibility with experimental results [28]. In the present study, the value of $C_{D}$ is equal to 2.0. Lift coefficient $C_{L O}$ is assumed to be equal to 0.3 [28]. Given that $q(x, t)$ is lift reduced coefficient function, the behaviour of the wake region can be expressed as [28]:

$\frac{\partial^{2} \bar{q}(x, t)}{\partial t^{2}}+\delta \omega_{s}\left[\bar{q}(x, t)^{2}-1\right] \frac{\partial \bar{q}(x, t)}{\partial t}+\omega_{s}^{2} \bar{q}(x, t)=F_{d}$

where $F_{d}$ is the force applied to the structure by the fluid, and $\delta$ is the fluid flow damping coefficient which depends on the average drag coefficient and is usually equal to 0.3 [30]. The vortex shedding frequency $\omega_{s}$ depends on the fluid velocity and Strouhal number:

$\omega_{s}=2 \pi S_{t} \frac{U}{D}$

where $S_{t}$ is the Strouhal number and dependent on the Reynolds numbers range and geometry of the cylinder's cross-section, the value chosen in this study is 0.2 [30]. The effect of the vortex-induced vibration, which is represented by $q(x, t)$ is usually considered proportional to the amplitude. According to the theory provided by Facchinetti et al. [28], the equation showing the best agreement with experimental results is as follows:

$F_{d}=\frac{P}{D} \frac{\partial^{2} w(x, t)}{\partial t^{2}}$

where $P=12$ which is calculated from experimental data fitting [28].

Having $m_{T D M}, K$ and $C$ as mass, stiffness and damping of the TMD vibration absorber, using Newton's second law, the equation of TMD vibration absorber which is shown in Fig. 1, is obtained as follows:

$m_{T D M} \frac{d^{2} Z(t)}{d t^{2}}+R(t)=0$

$R(t)=C\left[\frac{d Z(t)}{d t}-\frac{\partial w(x, t)}{\partial t}\right]+K[Z(t)-w(d, t)]$

Considering Eqs. (11)-(12) and (17), the external force applied to the beam is expressed as follows:

$f(x, t)=f_{D}(x, t)+f_{L}(x, t)+R(t) \delta(x-d)$

where $\delta(x-d)$ is Dirac delta function.

Dimensionless variables are defined as:

$\eta=\frac{w}{l} \quad \xi=\frac{x}{l} \quad \xi_{T D M}=\frac{d}{l}$

$\tau=\frac{1}{l^{2}} \sqrt{\frac{E I}{\rho A}} t \quad u=U l \sqrt{\frac{\rho A}{E I}} \Omega=\omega \sqrt{\frac{\rho A l^{4}}{E I}}$

$\Omega_{s}=\omega_{s} \sqrt{\frac{\rho A l^{4}}{E I}}=\Omega_{0 s} u \Omega_{0 s}=\frac{2 \pi l S_{t}}{D}$

$c_{L}=\frac{C_{L} \rho_{f} D l}{4 \rho A} \mu=\sqrt{\frac{\eta^{* 2} I}{l^{4} E \rho A}} \gamma=\sqrt{\frac{\eta^{* 2} A}{E \rho I}}$

$c_{D}=\frac{C_{D} \rho_{f} D l^{2}}{2 \rho A} \quad c=\frac{C l^{2}}{\sqrt{E I \rho A}} k=\frac{K l^{3}}{E I} \quad \beta=\frac{m_{T D M}}{\rho A l} \delta=\frac{P l}{D}$

where $\Omega$ indicates dimensionless natural frequency and the parameter $\varepsilon$ is used to demonstrate extremely small parameters. Utilizing the defined dimensionless vari- 
ables, the governing differential equation and the corresponding boundary conditions can be expressed as the following form:

$$
\begin{aligned}
& \mu \frac{\partial^{5} \eta}{\partial \xi^{4} \partial \tau}+\frac{\partial^{4} \eta}{\partial \xi^{4}}+\frac{\partial^{2} \eta}{\partial \tau^{2}} \\
& =\left[c\left(\frac{d z}{d \tau}-\frac{\partial \eta}{\partial \tau}\right)+k\left[z-\eta\left(\xi_{T D M}, \tau\right)\right] \delta\left(\xi-\xi_{T D M}\right)+\right. \\
& +c_{L} u^{2} q-c_{D} u \frac{\partial \eta}{\partial \tau}+\frac{3}{2} r\left(\frac{\partial \eta}{\partial \xi}\right)^{2} \frac{\partial^{2} \eta}{\partial \xi^{2}} \\
& +\gamma\left[2 \frac{\partial^{2} \eta}{\partial \xi^{2}} \frac{\partial \eta}{\partial \xi} \frac{\partial^{2} \eta}{\partial \xi \partial \tau}+\left(\frac{\partial \eta}{\partial \xi}\right)^{2} \frac{\partial^{3} \eta}{\partial \xi^{2} \partial \tau}\right] \\
& \beta \frac{d^{2} z}{d \tau^{2}}+c\left(\frac{d z}{d \tau}-\frac{\partial \eta}{\partial \tau}\right)+k\left[z-\eta\left(\xi_{T D M}, \tau\right)\right]=0 \\
& \frac{\partial^{2} q}{\partial \tau^{2}}+\lambda \Omega_{s}\left(q^{2}-1\right) \frac{\partial q}{\partial \tau}+\Omega_{s}^{2} q=\delta \frac{\partial^{2} \eta}{\partial \tau^{2}} \\
& \left.\eta\right|_{\xi=0}=\left.\frac{d^{2} \eta}{d \xi^{2}}\right|_{\xi=0}=\left.0 \quad \eta\right|_{\xi=1}=\left.\frac{d^{2} \eta}{d \xi^{2}}\right|_{\tilde{\xi}=1}=0
\end{aligned}
$$

In the present study, the nonlinear PDEs presented in Eq. (20) are discretized using the Galerkin method. Accordingly, the solution of the differential equation is considered as follows:

$$
\eta(\xi, \tau)=\sum_{n=1}^{N} \varphi_{n}(\xi) y_{n}(\tau)
$$

where $\varphi_{n}(\xi)$ and $V_{n}(T)$, represent the vibration mode shapes and generalized coordinates, respectively. The mode shape functions should satisfy all the boundary conditions of the system. Accordingly, in the present study, concerning the simple supports boundary conditions at the two ends, these functions are used as

$\varphi_{n}(\xi)=\sqrt{2} \sin (n \pi \xi)$

Considering the orthogonality of the special functions, the following conditions are satisfied:

$\int_{0}^{1} \varphi_{r}(\xi) \varphi_{s}(\xi) d \xi=\delta_{r s}$

$\int_{0}^{1} \varphi_{r}(\xi) \varphi^{\prime \prime \prime}(\xi) d \xi=\Omega_{r}^{2} \delta_{r s} \quad r, s=1,2, \ldots$.

where $\delta_{r s}$ is Kronecker delta function. Substituting the last equation in Eq. (20), and using the orthogonality of vibration mode shapes, the nonlinear governing equations are rewritten as:

$$
\begin{aligned}
& \sum_{n=1}^{N}\left[\Omega_{n}^{2}\left(\mu \dot{y}_{n}(\tau)+y_{n}(\tau)\right) \varphi_{n}(\xi)+\varphi_{n}(\xi) \ddot{y}_{n}(\tau)\right]+ \\
& +\left\{c\left[\sum_{n=1}^{N} \varphi_{n}(\xi) \dot{y}_{n}(\tau)-\frac{d z(\tau)}{d \tau}\right]+\right. \\
& \left.\quad+k\left[\sum_{n=1}^{N} \varphi_{n}(\xi) y_{n}(\tau)-z(\tau)\right]\right\} \delta\left(\xi-\xi_{a}\right)+ \\
& \quad+c_{L} u^{2} q-c_{D} u \sum_{n=1}^{N} \varphi_{n}(\xi) \dot{y}_{n}(\tau)+
\end{aligned}
$$

$$
\begin{aligned}
& +\frac{3}{2} r\left(\sum_{n=1}^{N} \varphi_{n}(\xi) \dot{y}_{n}(\tau)\right) \sum_{n=1}^{N} \varphi_{n}^{\prime \prime}(\xi) y_{n}(\tau)+ \\
& +\gamma\left[2 \sum_{n=1}^{N} \varphi_{n}^{\prime \prime}(\xi) y_{n}(\tau) \sum_{n=1}^{N} \varphi_{n}^{\prime}(\xi) y_{n}(\tau) \sum_{n=1}^{N} \varphi_{n}^{\prime}(\xi) \dot{y}_{n}(\tau)+\right. \\
& \left.+\left(\sum_{n=1}^{N} \varphi_{n}^{\prime}(\xi) y_{n}(\tau)\right) \sum_{n=1}^{N} \varphi_{n}^{\prime \prime \prime}(\xi) \dot{y}_{n}(\tau)\right]=0 \\
& \beta \frac{d^{2} z}{d \tau^{2}}+c\left(\frac{d z}{d \tau}-\sum_{n=1}^{N} \varphi_{n}(\xi) \dot{y}_{n}(\tau)\right)+ \\
& +k\left[z-\sum_{n=1}^{N} \varphi_{n}\left(\xi_{T D M}\right) y_{n}(\tau)\right]=0 \\
& \frac{\partial^{2} q}{\partial \tau^{2}}+\lambda \Omega_{0 s} u\left(q^{2}-1\right) \frac{\partial q}{\partial \tau}+\Omega_{0 s}^{2} u^{2} q=\delta \sum_{n=1}^{N} \varphi_{n}(\xi) \ddot{y}_{n}(\tau)
\end{aligned}
$$

By applying the Galerkin method for Eq. (24), the following equations with ordinary derivatives are obtained:

$\ddot{q}_{n}+\sum_{i=1}^{N}\left(B_{n i} \dot{q}_{i}+C_{n i} q_{i}\right)=\sum_{i=1}^{N} F_{i} f(\tau)+R_{n}(\tau)$

By defining vectors of the generalized variables $\mathbf{q}=\left[q_{1}, q_{2}, \ldots, q_{N}\right]^{\top}$, the Eq. (27) can be expressed in the following matrix form:

$$
\ddot{q}+B \dot{q}+C q=F f(\tau)+R(\tau)
$$

The coefficients of Eq. (25), which are the elements of the matrices appearing in Eq. (26), are determined using the following equation:

$$
\begin{aligned}
& B_{n i}=\int_{0}^{1} \varphi_{i}^{(4)} \varphi_{n} d \xi=\varepsilon \alpha C_{n i} \\
& R_{n}=\frac{3}{2} \lambda \int_{0}^{1}\left(\sum_{i=1}^{N} \varphi_{i}^{\prime} q_{i} \sum_{j=1}^{N} \varphi_{j}^{\prime} q_{j}\right) \varphi_{n} \mathrm{~d} \xi+ \\
& +\varepsilon \mu \int_{0}^{1}\left(2 \sum_{i=1}^{N} \varphi_{i}^{\prime \prime} q_{i} \sum_{j=1}^{N} \varphi_{j}^{\prime} q_{j} \sum_{k=1}^{N} \varphi_{k}^{\prime} q_{k}+\sum_{i=1}^{N} \varphi_{i}^{\prime} q_{i} \sum_{j=1}^{N} \varphi_{j}^{\prime} q_{j} \sum_{k=1}^{N} \varphi_{k}^{\prime \prime} q_{k}\right) \varphi_{n} \mathrm{~d} \xi
\end{aligned}
$$

Considering the first ten mode shapes in Galerkin method, i.e. $N=10$, the differential equations with ordinary derivatives obtained in Eq. (24), appear as 13 coupled nonlinear differential equations. Runge-Kutta numerical method is applied to solve these equations, and in the next section, the results of the numerical solution will be discussed.

\section{RESULTS}

There has been no study conducted to investigate the effects of vibration absorber on the fluid-inductive vibration behaviour of viscoelastic beams. Therefore, in order to verify the results of the presented model, by ignoring the impact of external fluid flow and vibration absorber damping, i.e., $q=c=0$, a similar system of simply supported beam studied by Rossi et al. [31] is taken into account.

In Table 1, the first two natural frequencies of the system and the natural frequency of the TMD absorber $\Omega_{\text {Abs }}$ are compared with the corresponding results of [31] for different dimensionless mass and stiffness parameters of the absorber. As can be seen, there is an acceptable agreement with the results of [31], and the maximum er- 
Table 1: Comparison the natural frequencies of the beam with undamped vibration absorber and the results of Ref. [31]

\begin{tabular}{|c|c|c|c|c|c|c|c|c|c|c|}
\hline & $\beta$ & \multicolumn{3}{|c|}{$\Omega_{\text {Abs }}$} & \multicolumn{3}{|c|}{$\Omega_{2}$} & \multicolumn{3}{|c|}{$\Omega_{3}$} \\
\hline \multirow[t]{2}{*}{---} & & Present & Ref. [30] & $\begin{array}{c}\text { Error } \\
(\%)\end{array}$ & Present & Ref. [30] & $\begin{array}{c}\text { Error } \\
(\%)\end{array}$ & Present & Ref. [30] & $\begin{array}{c}\text { Error } \\
(\%)\end{array}$ \\
\hline & 0 & - & - & - & 9.83 & 9.67 & 1.32 & 88.56 & 88.26 & 0.37 \\
\hline \multirow{3}{*}{$k=1$} & 0.2 & 2.23 & 2.21 & 0.91 & 0.91 & 9.98 & 0.50 & 88.54 & 88.84 & 0.34 \\
\hline & 1 & 1.00 & 0.99 & 1.01 & 1.01 & 9.97 & 0.69 & 88.54 & 88.84 & 0.34 \\
\hline & 3 & 0.60 & 0.57 & 3.51 & 3.51 & 9.97 & 0.69 & 88.54 & 88.84 & 0.34 \\
\hline \multirow{3}{*}{$k=100$} & 0.2 & 8.15 & 8.16 & 0.12 & 0.12 & 26.62 & 0.95 & 89.86 & 90.04 & 0.20 \\
\hline & 1 & 5.02 & 5.11 & 1.76 & 1.76 & 19.03 & 1.01 & 89.82 & 89.98 & 0.18 \\
\hline & 3 & 3.14 & 3.17 & 0.95 & 0.95 & 17.71 & 0.78 & 89.34 & 89.97 & 0.71 \\
\hline \multirow{3}{*}{$k=1000$} & 0.2 & 8.56 & 8.32 & 2.88 & 2.88 & 66.01 & 0.96 & 110.23 & 109.95 & -0.25 \\
\hline & 1 & 5.68 & 5.62 & 1.07 & 1.07 & 46.98 & 1.47 & 102.98 & 102.45 & -0.51 \\
\hline & 3 & 3.77 & 3.65 & 3.28 & 3.28 & 42.18 & 1.24 & 102.36 & 101.53 & -0.81 \\
\hline
\end{tabular}

ror of the results is about $3.5 \%$. The results show that increasing the TMD absorber mass causes a decrease in the natural frequencies of the beam. On the other hand, increasing the TMD absorber rigidity increases the natural frequency of the beam, due to the increase in the equivalent stiffness of the structure. Using this effect of TMD vibrating absorber on vibration characteristics of the beam, it is possible to reduce the impacts of fluid-induced vibration. Hence, resonances caused by flu-

with TMD vibration absorber

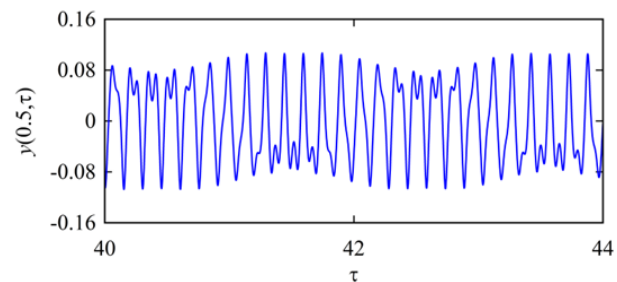

(a)

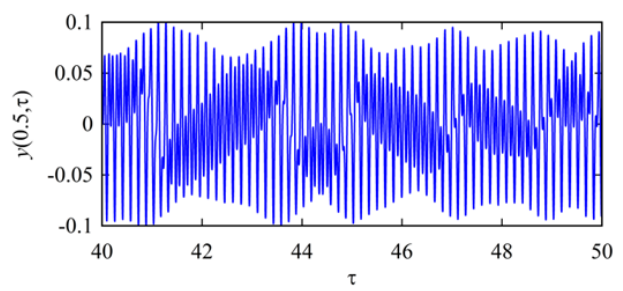

(b)
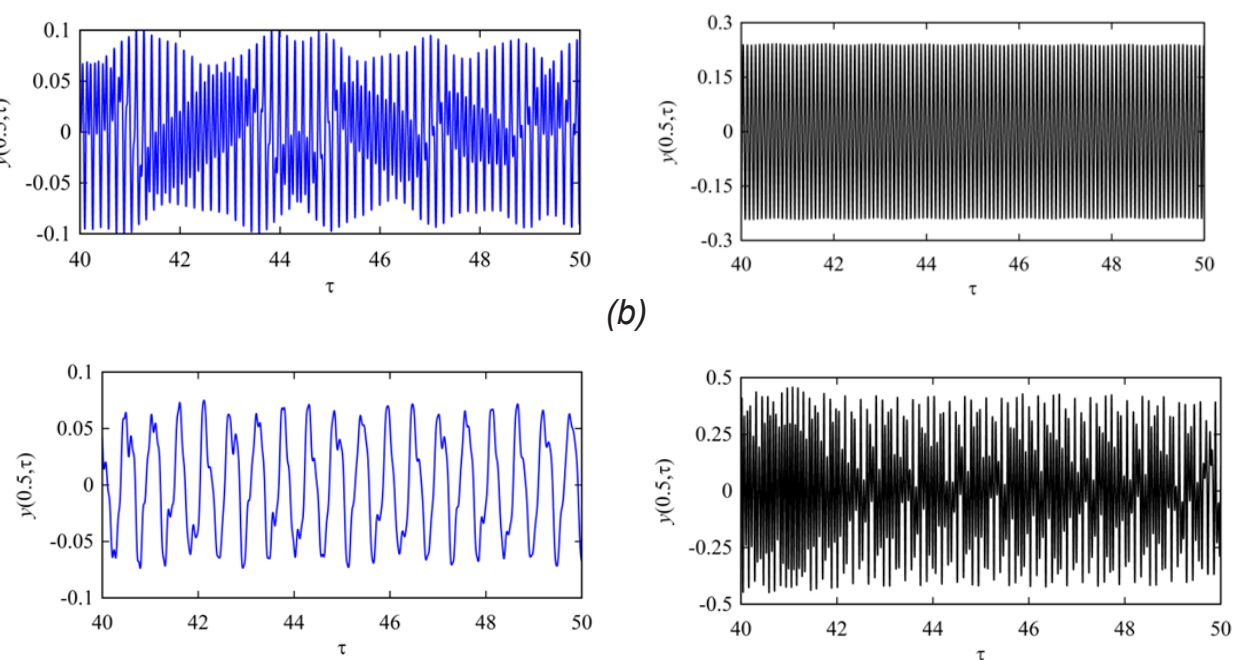

(c)

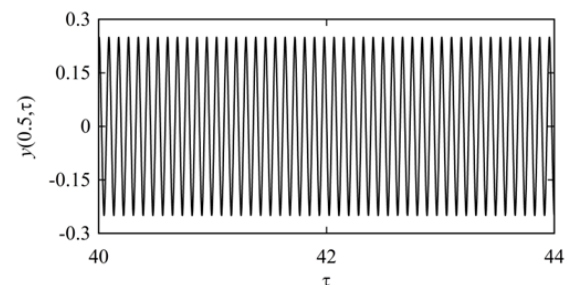

id-induced vibration are avoided by changing the Lock-in range.

Fig. 4 shows the dynamic response at the midpoint of the viscoelastic beam for different external flow dimensionless velocities with (and without) TMD absorber having properties $c=0,5, k=100$ and $\beta=0,3$. As shown in Fig. $4 \mathrm{a}$, in the absence of external fluid flow, the response of the system is oscillating, and in the presence of the vibration absorber and in its absence, the dimensionless without TMD vibration absorber 
amplitude is 0.084 and 0.22 , respectively. In this case, the effect of fluid inertia can be neglected, and only the effect of the fluid mass in reducing the natural frequency of the beam is taken into account. At low fluid velocities, which corresponds to small Reynolds and Strouhal numbers, by increasing the fluid velocity, Von Karman vortex streets are created symmetrically behind the beam due to negative pressure. This applies lift and drag forces to the beam, which increases the amplitude of the vibrations (Fig. 4b). Moreover, the results show that the use of TMD vibration absorber in structures which are affected by fluid-induced vibrations causes a significant reduction in the system oscillation amplitude; For instance, at fluid velocities $u=0, u=0,5$ and $u=1$ oscillation amplitude of the system is decreased by $47 \%, 41 \%$ and $86 \%$, respectively.

Vibration absorber reduces the maximum amplitude of the system oscillations. For this purpose, by defining the parameter in Eq. (28), which represents the percentage of decrease in the amplitude of the system oscillations, the effect of vibration absorber on the behaviour of viscoelastic beam is investigated.

$$
A R R=\frac{y_{\max }^{0}(0.5, \tau)-y_{\max }(0.5, \tau)}{y_{\text {max }}^{0}(0.5, \tau)} \times 100
$$

where $y_{\text {max }}^{0}(0,5, T)$ and $y_{\max }(0,5, T)$ indicate the maximum amplitude of the beam oscillation in the absence and presence of TMD vibration absorber.

In order to study the effect of vibration absorber on the system performance, Fig. 5 shows a comparison between the vibration response of the beam in the presence and absence of the TMD vibrating absorber for different values of the absorber stiffness parameter. Here, with the constant values $u=0$ and $\varepsilon \alpha=5 \times 10^{-3}$, and also absorber parameters $\mu=0,2$ and $c=0,5$, the effect of the absorber stiffness has been studied. As can be seen, the presence of the TMD vibration absorber significantly reduces the amplitude of the system oscillations. The decrease depends on the absorber parameters; by increasing $k$, the effect of absorber on decreasing the amplitude

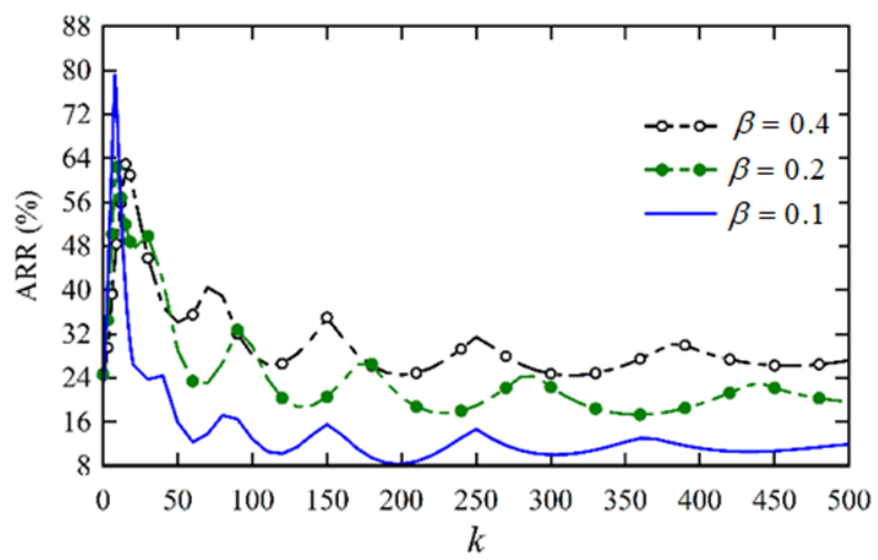

Figure 5: Comparison between the vibration response of the viscoelastic beam in the presence and absence of the TMD vibration absorber for different values of the absorber stiffness parameter of the oscillations is lowered. As an example, for $k=500$ and $k=1$, the decrease in the amplitude of the system oscillation are $13.6 \%$ and $31.5 \%$, respectively. According to the results, it is observed that absorber with stiffness $k=10$, which reduces the amplitude of system oscillations by $75 \%$, has the best performance. Considering that in this case, the dimensionless natural frequency of the absorber $\Omega_{a b s}=\sqrt{ } k / \mu=7,07$ is near the natural frequency of the beam with $u=2$, the TMD system absorbs the highest amount of energy, and reduction of the amplitude of the oscillations has the maximum value.

In order to investigate the dynamic behaviour of the viscoelastic beam with TMD vibration absorber under the fluid flow excitation, the frequency response of the system is extracted in the steady-state. Fig. 6 demonstrates the maximum amplitude of the system oscillations with vortex shedding frequency for different values of absorbent dimensionless mass. As the results show, the jump phenomenon occurs due to the nonlinear behaviour of the system. In the absence of the TMD vibration absorb-

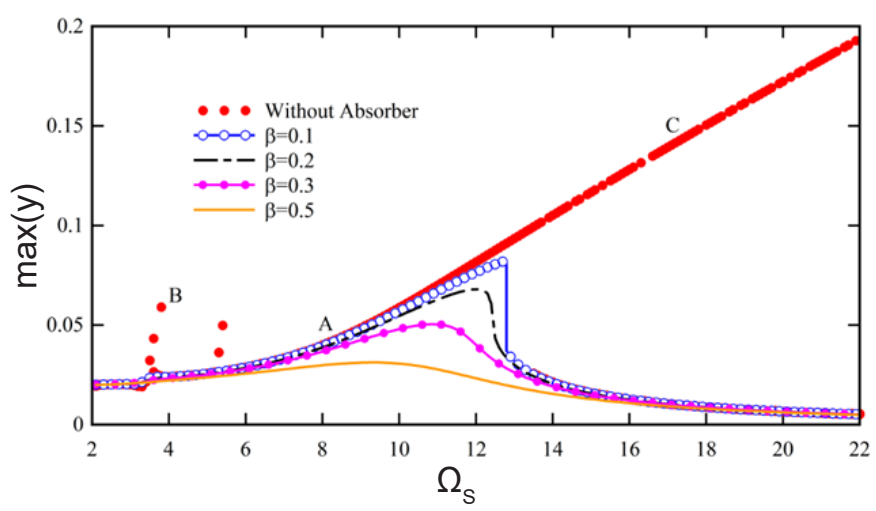

Figure 6: The maximum amplitude of the system oscillations with vortex shedding frequency for different values of absorber dimensionless mass

er, increasing the parameter $\Omega_{S}$, resonance occurs in the system. Resonances occur at $\Omega_{S}$ about 3.6 and 5.3, and when it reaches 13.5, jump phenomenon occurs, and the system behaviour becomes unstable. Another result from Fig. 6 is that the use of vibration absorber has a significant effect on the frequency-response curve of the system, and by increasing the absorber dimensionless mass parameter, jump phenomenon is eliminated from the system behaviour. For $\beta=0,1$, jump phenomenon is seen at $\Omega_{S}=12,3$; increasing the $\Omega_{S}$ maximum amplitude of the oscillations is sharply reduced, and the resonance regions omitted from the system behaviour. These results show the useful ability of the presented method to control vibrations in viscoelastic beams affected by external fluid flow.

To study the effect of fluid flow on the dynamic response of the system in stable and unstable conditions, the steady-state time response and also phase and Poincaré diagrams for the viscoelastic beam without vibration absorber are shown in Figs. 7 to 9. In Fig. 7, which is plotted for the conditions of the point A (see in Fig. 6), the vibration amplitude is stable, and the time response 
is periodic so that the Poincare map shows a point in the phase plane. Near point $B$, subharmonic resonances occur in the system, the vibration amplitude is unstable and has a semi-periodic cycle which is visible in Fig. 8. Such a behaviour is also observed for the system in the region $\mathrm{C}$ in Fig. 9.

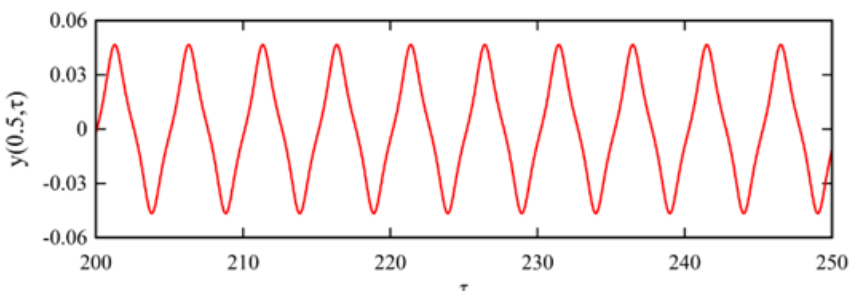

(a)

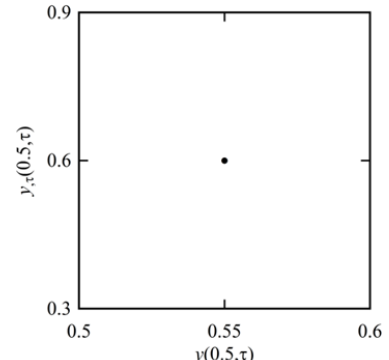

(b)

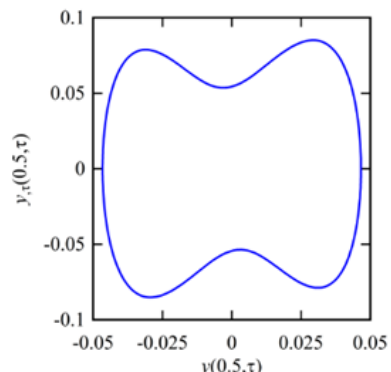

(c)

Figure 7: Periodic time response of the system for the point a conditions

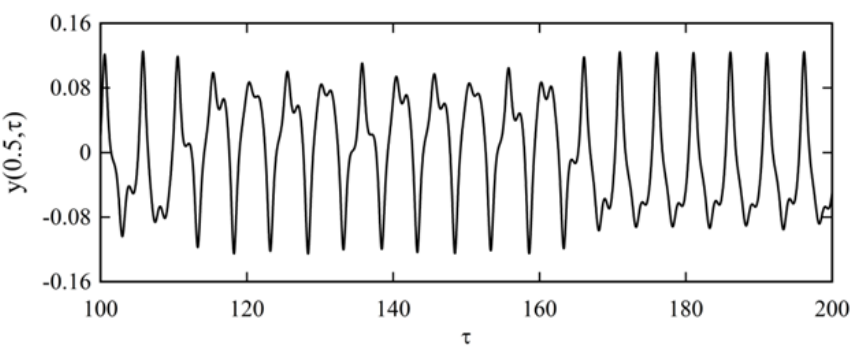

(a)

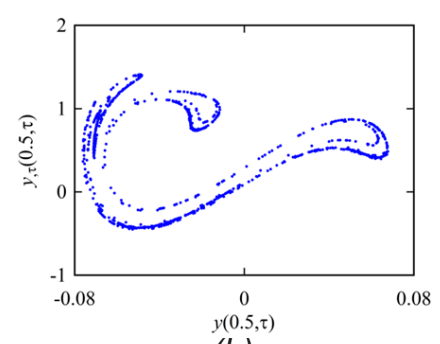

(b)

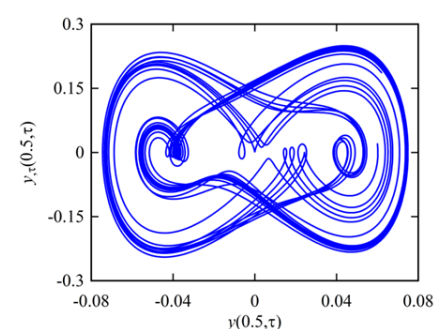

(c)
Figure 8: Semi-periodic time response of the system for the point $B$ conditions

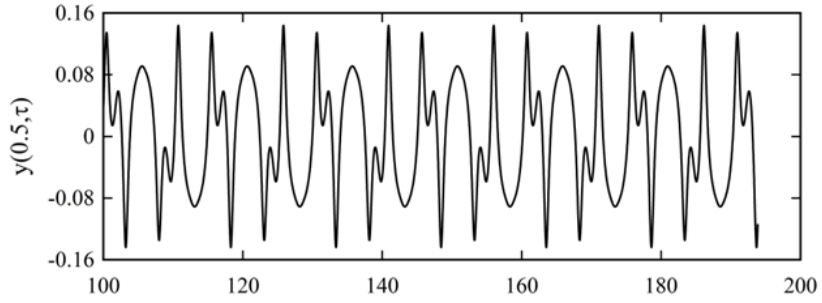

(a)

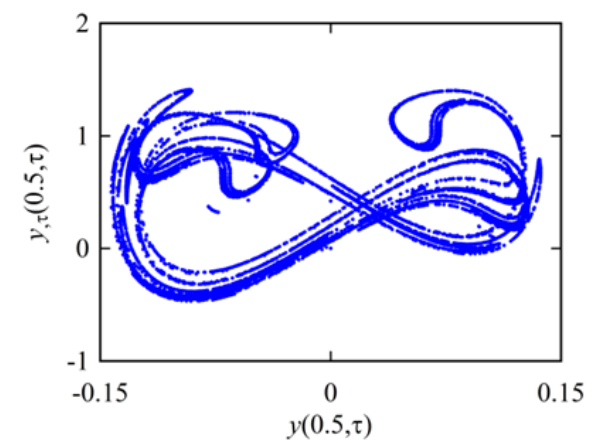

(b)

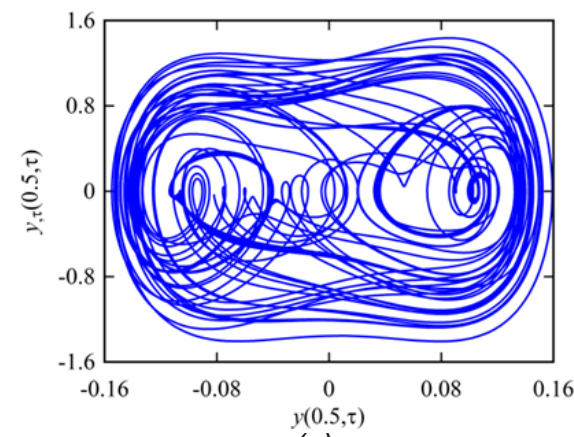

(c)

Figure 9: Chaotic time response of the system for the point $\mathrm{C}$ conditions

\section{CONCLUSION}

In this study, the ability to utilize mass-spring-damper vibration absorber focused on controlling fluid-induced vibration in viscoelastic beams was semi-analytically investigated. Nonlinear equations governing the motion were derived by taking into account the effects of nonlinear strains and were discretized by Galerkin's method. The results show that applying the vibration absorber significantly reduces the amplitude of the system oscillations, i.e., the maximum amplitude is decreased at fluid velocities $u=0, u=0,5$ and $u=1$ by $47 \%, 41 \%$ and $86 \%$, respectively. Concerning the influences of the TMD system stiffness on the responses of the structure, the results showed that increasing the stiffness of TMD; the energy pumping effect can be enhanced with the presence of stable responses, thereby reducing the vibration amplitudes of the beam. This results in a significant reduction in the fluid-induced vibration amplitudes of the viscoelastic beam for appropriate TMD vibration absorber parameter values. Results of the current study show the proper ability to use passive tuned mass damper absorbers to control fluid-induced vibrations in structures. 


\section{REFERENCES}

1. Besem, F.M., Kamrass, J.D., Thomas, J.P., Tang, D., and Kielb, R.E. (2016). Vortex-induced vibration and frequency lock-in of an airfoil at high angles of attack. Journal of Fluids Engineering, vol. 138,12-34.

2. Purohit, A., Darpe, A.K., Singh, S. (2016). Experimental investigations on flow-induced vibration of an externally excited flexible plate. Journal of Sound and Vibration, vol. 371, 237-251.

3. Rose, J.B.R., and Jinu, G. (2016). Influence of aeroelastic flow-induced oscillations on the fatigue life of an airplane wing structure. International Journal of Modelling, Identification and Control, vol. 25, 199216.

4. Liangjie, M., Qingyou, L., Shouwei, Z., JIANG, W., Zhengli, L., and Tao, P. (2015). Vortex-induced vibration mechanism of drilling riser under shear flow. Petroleum Exploration and Development, vol. 42, 112-118.

5. Song, L., Fu, S., Cao, J., Ma, L., and Wu, J. (2016). An investigation into the hydrodynamics of a flexible riser undergoing vortex-induced vibration. Journal of Fluids and Structures, vol. 63, 325-350.

6. Lu, Z., Fu, S., Zhang, M., and Ren, H. (2019). An efficient time-domain prediction model for vortex-induced vibration of flexible risers under unsteady flows. Marine Structures, vol. 64, 492-519.

7. Zhang, M., Fu, S., Song, L., Tang, X., and He, Y. (2018). A time domain prediction method for the vortex-induced vibrations of a flexible riser. Marine Structure, vol. 458, 59-81.

8. Tatiana, P., Dario, B., and Gianmarco, V. (2018). Vibration analysis for the comfort assessment of a superyacht under hydrodynamic loads due to mechanical propulsion. Ocean Engineering, vol. 155, 310-323.

9. Jamshidi-Naeini, Y., Bavil, A.K., Egal, A., and Oldewage-Theron, W. (2019). Hemoglobin and ferritin concentrations are positively associated with blood pressure and hypertension risk in older adults: a retrospective cross-sectional study, Sharpeville, South Africa. Asia Pacific journal of clinical nutrition.vol. 28, no. 3, 533 .

10. Estlack, Z., Bavil, A.K., Kim, J.(2020). Design, Fabrication. Biomimetic Microengineering, CRC publication, vol. 16, 141.

11. Wang, J., Fu, S., Baarholm, R., Wu, J., and Larsen, C.M. (2015). Fatigue damage induced by vortex-induced vibrations in oscillatory flow. Marine Structures, vol. 40, 73-91.

12. Han, Q., Ma, Y., Xu, W., and Zhang, S. (2018). An experimental study on the hydrodynamic features of two side-by-side flexible cylinders undergoing flow-induced vibrations in a uniform flow. Marine Structures, vol. 61, 326-342.
13. Bavil, A. K., \& Razavi, S. E. (2017). On the thermo-flow behavior in a rectangular channel with skewed circular ribs. Mechanics \& Industry, 18(2), 225.

14. Xu, W., Cheng, A., Ma, Y., and Gao, X. (2018). Multimode flow-induced vibrations of two side-by-side slender flexible cylinders in a uniform flow. Marine Structures, vol. 57, 219-236.

15. Tan, J., Jiang, J., Liu, M., Feng, Q., Zhang, P., and Ho, S.C.M. (2019). Implementation of Shape Memory Alloy Sponge as Energy Dissipating Material on Pounding Tuned Mass Damper: An Experimental Investigation. Appl. Sci, vol. 9, 1079.

16. Wu, X., Ge, F., and Hong, Y. (2012). A review of recent studies on vortex-induced vibrations of long slender cylinders. Journal of Fluids and Structures, vol. 28, 292-308.

17. Kasiri Ghahi, M., and Sanaeirad, A. (2018). Vibration design for horseshoe shaped tunnel by Abaqus software, Uct Journal of Research in 17 Science, Engineering and Technology, vol. 02, 7-12.

18. Bourguet, R., Karniadakis, G.E., and Triantafyllou, M.S. (2011). Lock-in of the vortex-induced vibrations of a long tensioned beam in shear flow. Journal of Fluids and Structures, vol. 27, 838-847.

19. Bourguet, R., Karniadakis, G.E., and Triantafyllou, M.S. (2013). Multi-frequency vortex-induced vibrations of a long tensioned beam in linear and exponential shear flows. Journal of Fluids and Structures, vol. 41, 33-42.

20. Bourguet, R., Karniadakis, G.E., and Triantafyllou, M.S. (2013). Phasing mechanisms between the inline and cross-flow vortex-induced vibrations of a long tensioned beam in shear flow. Computers \& Structures, vol. 122, 155-163.

21. Daniels, S.J., Castro, I.P., and Xie, Z.T. (2016). Numerical analysis of freestream turbulence effects on the vortex-induced vibrations of a rectangular cylinder. Journal of Wind Engineering and Industrial Aerodynamics, vol. 153, 13-25.

22. Marra, A.M., Mannini, C., and Bartoli, G. (2015). Measurements and improved model of vortex-induced vibration for an elongated rectangular cylinder. Journal of Wind Engineering and Industrial Aerodynamics, vol. 147, 358-367.

23. Han, X., Lin, W., Tang, Y., Zhao, C., and Sammut, K. (2015). Effects of natural frequency ratio on vortex-induced vibration of a cylindrical structure. Computers \& Fluids, vol. 110, 62-76.

24. Jiang, X., Andreopoulos, Y., Lee, T., and Wang, Z. (2016). Numerical investigations on the vortex-induced vibration of moving square cylinder by using incompressible lattice Boltzmann method. Computers \& Fluids, vol. 24, 270-277. 
25. Wang, W., Wang, X., Hua, X., Song, G., and Chen, Z. (2018). Vibration control of vortex-induced vibrations of a bridge deck by a single-side pounding tuned mass damper. Engineering Structures, vol. 173, 61-75.

26. Chen, J.E., He. W., Zhang, W., Yao, M.H., Liu, J., and Sun, M. (2018). Vibration suppression and higher branch responses of beam with parallel nonlinear energy sinks. Nonlinear Dynamics, vol. 91, 885-904.

27. Tatiana, P., Dario, B. (2017). Developments of Tuned Mass Damper for yacht structures. Ocean Engineering, vol. 141, 249-264.

28. Facchinetti, M.L., De Langre, E., and Biolley, F. (2020). Coupling of structure and wake oscillators in vortex-induced vibrations. Journal of Fluids and structures, vol. 19, 123-140.
29. Keber, M., and Wiercigroch, M.(2018). Dynamics of a vertical riser with weak structural nonlinearity excited by wakes. Journal of Sound and Vibration, vol. 315, 685-699.

30. Chen, S.S. (1996). Flow-induced vibration of circular cylindrical structures. Argonne National Lab. (ANL), Argonne, IL (United States).

31. Rossi, R.E., Laura, P.A.A., Avalos, D.R., and Larrondo, H. (2013). Free Vibrations of Timoshenko Beams Carrying Elastically Mounted, Concentrated Masses. Journal of Sound and Vibration, vol. 165, 209-223. 